\title{
Study of clinical profile of megaloblastic anemia: An experience of six year at Kathmandu University Hospital, Dhulikhel
}

\author{
B.R. Pokharel ${ }^{1}$, P. Pant ${ }^{2}$, R. Gurung, ${ }^{3,}$ R. Koju ${ }^{4}$, T.R.S. Bedi ${ }^{5}$, R. Makaju ${ }^{6}$, S. Sanjel ${ }^{7}$ \\ Lecturer $^{1-3}$, Assistant Professor ${ }^{4}$, Professor ${ }^{5}$, Department of Medicine, Assistant professor Pathology ${ }^{6}$, Lecturer Community \\ Medicine ${ }^{7}$, Kathmandu University Hospital, Dhulikhel
}

\begin{abstract}
Megaloblastic anemia is not uncommon, however varied of presentation makes difficult to come to conclusion. It's more difficult when resources are limited. In this back ground clinical features of megaloblastic anemia are very important for diagnosis and treatment. It is a retrospective analysis of data from December 2003 to September 2009. During the period, bone marrow aspiration was done for analysis of cause and type of anemia.A total of 14 cases bone marrow findings was consistent with megaloblastic anemia. These reports were taken into consideration after pathologist impression. Serum Vitamin B12 and $\mathrm{RBC}$ folate were not done in view of limited resources and financial constrain. In the analysis 14 $(11.96 \%)$ bone marrow findings were consistent with megaloblastic anemia. Regarding clinical presentation; pallor was $13(92.85 \%)$ followed by glossitis $5(35.71 \%)$ and Jaundice $5(35.71 \%)$, paresthesia $3(21.42 \%)$, and pigmentation, ataxia, confusion each case $(7.14 \%)$. Mostly the patients were between the 21- 40 years of age. Megloblastic anemia is common clinical problem with pallor and glossitis as major presentation. However some had neurological features. This disease can be diagnosed and treated on the basis of bone marrow aspiration when resources are limited.
\end{abstract}

Key words: Ataxia, bone marrow aspiration, megaloblastic anemia, paresthesias.

\section{Introduction}

The megaloblastic anemia is a common disease with varied presentation. In the setting like ours this kind of study will help to recognize the megloblastic anemia clinically. The megaloblast, the morphologic hallmark of the syndrome, is a product of impaired DNA formation which in turn is due to deficiencies of vitamin B12 (cobalamin, Cbl) or folic acid (FA) ${ }^{1,2}$ This study was carried to find out Correspondence: B. R. Pokhrel E-mail: drbrpokh@gmail.com common clinical presentation of megaloblastic anemia in patients attending Dhulikhel hospital, Kathmandu University hospital.

\section{Meterials and methods}

A retrospective observational study was conducted from data of patient who presented with anemia from December 2003 to September 2009 at the Department of Medicine, Dhulikhel hospital, Kathmandu University hospital, Kavre, Nepal. The 
ethical approval was taken from ethical committee. This study was conducted with patient above 15 years without concurrent diseases like renal failure, liver diseases or malignancy. Bone marrow aspiration was performed using Klima needle from posterior superior illac spine in all cases. These findings were taken into consideration after pathologists reports. A full blood counts was done on improved Neubeauer counting chamber and or hematology auto analyzer. Peripheral blood smear were also performed in each case. Wright and iron stain were performed on the bone marrow smears. The data was analyzed based on clinical history which included age, sex, address, .ethnicity and occupation. Clinical data was also done to find out the significance of pallor, glossitis, jaundice, pigmentation, paraesthesia, ataxia, bleeding, confusion, dementia and others. Data collections were done by retrieving the files from medical record department. Data analysis was done using SPSS VS 16.

\section{Results}

Bone marrow aspirations were done from Dec 2003 to Sep 2009 for evaluation anemia. In the analysis $14(11.96 \%)$ bone marrow findings were consistent with megaloblastic anemia. Regarding clinical presentation; pallor- 13 (92.85\%) followed by glossitis 5 (35.71\%) and Jaundice- 5 (35.71\%), paresthesia $3(21.42 \%)$, and pigmentation, ataxia, confusion each case $(7.14 \%)$. Six out of 14 patients were between- 21 - 40 years of age. Clinical data is shown in table 1 and a clinical profile of megaloblastic anemia is shown in table 2. Age distribution is shown in figure 1.

\section{Table 1.Clinical data of Megaloblastic anemia}

\begin{tabular}{|c|c|c|c|c|c|c|c|c|c|}
\hline $\mathrm{SN}$ & Age/sex & Pallor & Glossitis & Jaundice & Pigmentation & Paresthesia & Ataxia & Confusion & Others \\
\hline 1 & 30/M & + & - & + & - & + & + & + & - \\
\hline 2 & 28/M & + & + & + & + & - & - & - & - \\
\hline 3 & $49 / \mathrm{F}$ & + & - & + & - & - & - & - & - \\
\hline 4 & $53 / \mathrm{M}$ & + & - & - & - & - & - & - & Weight loss \\
\hline 5 & $34 / \mathrm{M}$ & + & - & - & - & - & - & - & SOB/plapitation \\
\hline 6 & $28 / \mathrm{M}$ & + & + & + & - & - & - & - & Weakness \\
\hline 7 & $40 / \mathrm{M}$ & + & - & - & - & - & - & - & Weakness \\
\hline 8 & $50 / \mathrm{F}$ & + & + & - & - & - & - & - & SOB \\
\hline 9 & $59 / \mathrm{M}$ & + & - & - & - & - & - & & SOB/weakness \\
\hline 10 & 30/M & + & - & + & - & - & - & - & - \\
\hline 11 & $65 / \mathrm{M}$ & + & + & - & - & - & - & - & SOB \\
\hline 12 & $80 / \mathrm{F}$ & - & - & - & - & + & - & - & - \\
\hline 13 & $60 / \mathrm{M}$ & + & + & - & - & + & - & - & - \\
\hline 14 & 20/M & + & - & - & - & - & - & - & Weakness \\
\hline
\end{tabular}


B.R. Pokharel et al. Study of clinical profile of megaloblastic anemia.

Table 2. Clinical profiles of Megaloblastic anemia

\begin{tabular}{lcc}
\hline $\begin{array}{l}\text { Clinical } \\
\text { features }\end{array}$ & $\begin{array}{c}\text { Number } \\
\text { of cases }\end{array}$ & Percentage \\
\hline Pallor & 13 & 92.85 \\
Glossitis & 5 & 35.71 \\
Jaundice & 5 & 35.71 \\
Pigmentation & 1 & 7.14 \\
Paresthesia & 3 & 21.42 \\
Ataxia & 1 & 7.14 \\
Confusion & 1 & 7.14 \\
Others & 8 & 57.14 \\
\hline
\end{tabular}

Note: others-(weakness/shortness of breath/ palpitation/weight loss)

\section{Figure 1. Age distribution of megaloblastic} anemia

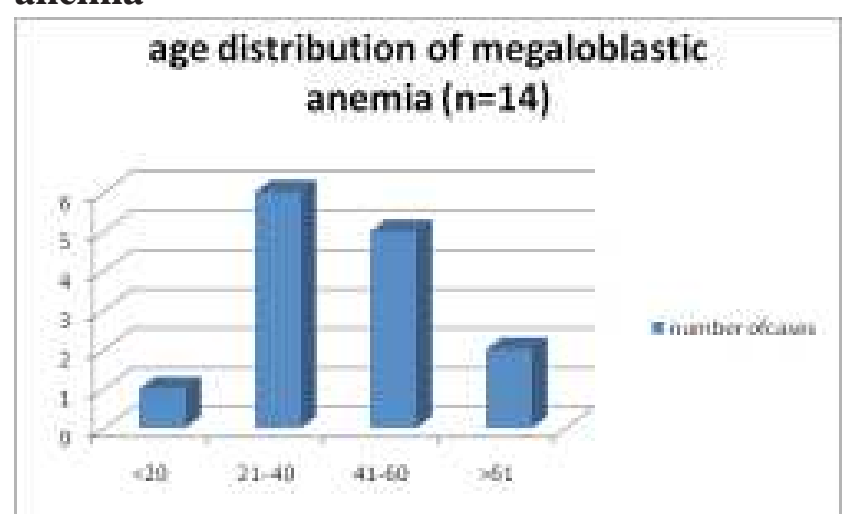

\section{Discussion}

Deficiency of either folic acid or vitamin B12 results in megaloblastic anaemia:wth the release of immature erythrocytes into the circulation erythrocytes due to a failure of the normal process of erythrocyte maturation in the bone marrow (Wickramasinghe, 1995, 1999). ${ }^{3}$
Symptoms of anemia like weakness, fatigue, exertional dyspnea, palpitations, dizziness and aches and pains all over the body are commonly present these symptoms are comparable with shah A. Megablastic anemia. ${ }^{4}$ Pallor was the most common clinical finding $92 \%$ followed by glossitis Which finding is comparable with the finding of Blood Cells, Molecules, and Diseases. ${ }^{5}$

Nearly $22 \%$ of the patient had peripheral neuropathy which is in contrast with findings mentioned in Blood Cells, Molecules, and Diseases only 7\%. Megalooblastic anemia due specifically to vitamin B12 deficiency, in which there is also spinal cord degeneration. The patient may have hard-to-characterize neuropsychiatric problems consisting of paresthesias, numbness, weakness, loss of dexterity, impaired memory, and personality changes Current concepts in the diagnosis of cobalamin deficiency. ${ }^{6}$

The common age of presentation of megaloblastic anemia in our centre was between the age of $21-$ 40 yrs which is in contradictory with following findings. Probably due to the life span in western countries is much longer than in Nepal Pernicious anemia is a leading cause of peripheral neuropathy. It is a disease of later life; only about $10 \%$ of patients are aged, 40 years; by the age of 60 years about $1 \%$ of the population are affected, rising to $2-5 \%$ of people aged 65 years, as a result of atrophic gastritis (commonly due to autoimmune disease) and hence impaired secretion of intrinsic factor, which is required for the absorption of vitamin B12 (Baik \& Russell, 1999). ${ }^{7}$

The prevalence of vitamin B12 deficiency, whether defined as low vitamin B12 or metabolically 
Journal of College of Medical Sciences-Nepal,2011, Vol-7,No-2

significant vitamin B12 deficiency increased with age in all above three studies, from about 1 in 20 among people aged 65-74 years to 1 in 10 or even greater among people aged 75 years or greater. The prevalence of folate deficiency also increased with age, and was similar to that for vitamin B12 deficiencies, Vitamin B12 and folate deficiency in later life. ${ }^{8}$

The fact may be related to the life expectancy of Nepali people is 61years according to $\mathrm{WHO}$, Mortality fact sheet; Nepal.9

Up to one-third of patients develop neurological signs without megaloblastosis, and high intakes of folate may prevent megaloblastosis in vitamin B12 deficiency (Dickinson, 1995; Savage \& Lindenbaum, 1995). ${ }^{10}$ Seizures and tremor were observed in $46.6 \%(7 / 15)$ and $33 \%(5 / 15)$ of patients, respectively as mentioned in the Neurologic findings of nutritional vitamin B12 deficiency in children $^{11}$. was in contrast of our findings where neither of the patient had seizure or tremor. problably due to the age group we selected.

Serum Vitamin B12 and RBC folate level were not done in view limited resources available and financial constraint. A better study with more sample size on multicentric basis is recommended to validate the finding of the study.

\section{Conclusions}

Megaloblastic anemia is not uncommon in our context. Pallor, glossitis, jaundice, paresthesia, pigmentation, ataxia, unconsciousness were presentation in our study. This study with summary of clinical feature being mentioned as above will definitely help clinician the for the diagnosis and treatment of megaloblastic anemia.

\section{References}

1. R.K. Pruthi, A. Tefferi. Pernicious anemia revisited. Mayo Clin Proc. 1994;69(2):144-50.

2. R.H. Allen, S.P. Stabler, D.G. Savage, Lindenbaum J. Diagnosis of cobalamin deficiency I: usefulness of serum methylmalonic acid and total homocysteine concentrations. Am J Hematol. 1990;34(2):90-8.

3. S.N. Wickramasinghe SN. Morphology, biology and biochemistry of cobalamin- and folate-deficient bone marrow cells. Baillieres Clin Haematol. 1995; 8(3):44159.

4. A. Shah. Megaloblastic anemia - Part II. Indian J. Med. Sci. 2004;58:309-11.

5. M.S. Harakati MS. Pernicious anemia in Arabs. Blood Cells Mol Dis. 1996;22(2):98-103. 6.

6. R. Green, L.J. Kinsella. Current concepts in the diagnosis of cobalamin deficiency. Neurology. 1995 ;45(8):1435-40.

7. H.W. Baik, R.M. Russell. Vitamin B12 deficiency in the elderly. Annu Rev Nutr. 1999;19:357-77.

8. R. Clarke, J. Grimley Evans, J. Schneede, et al. Vitamin B12 and folate deficiency in later life. Age Ageing. 2004 ;33(1):34-41.

9. World Health Organization. Mortality Country Fact Sheet 2006 Nepal.

10. C.J. Dickinson. Does folic acid harm people with vitamin B12 deficiency? QJM. 1995;88(5):357-64.

11. F. Incecik, M.O. Hergüner, S. Altunbasak, et al. Neurologic findings of nutritional vitamin B12 deficiency in children. Turk J Pediatr. 2010;52(1):17-21. 